\title{
Performance de six cultivars de tomates Lycopersicon esculentum Mills. contre la jaunisse en cuillère des feuilles, le flétrissement bactérien et les nématodes à galles.
}

\author{
Senan SoRO ${ }^{1,2 *}$, Mamadou DoumBIA ${ }^{2}$, Daouda DAO ${ }^{1}$, TSCHANNEN Andres $^{1} \&$ Olivier GIRARDIN $^{1}$. \\ ${ }^{1}$ Centre Suisse de Recherches Scientifiques, 01 BP 1303 Abidjan 01 (Côte d'Ivoire); \\ 2 Université de Abobo-Adjamé , UFR des Sciences de la Nature, 02 BP 801 Abidjan 02 (Côte d'Ivoire). \\ *Auteur pour les correspondances (E-mail : senan.soro@csrs.ci) \\ Reçu le 14-04-2006, accepté le 14-05-2007.
}

\begin{abstract}
Résumé
La tomate est largement produite en Côte d'Ivoire. Malgré cette importance, sa production est soumise à de nombreuses contraintes dont les principales sont entre autres : la jaunisse en cuillère des feuilles, le flétrissement bactérien et les nématodes à galles. En vue d'identifier des cultivars performants, une étude portant sur six cultivars améliorés de tomate a été conduite dans la région de Toumodi (Côte d'Ivoire). Elle s'est déroulée sur deux cycles de production, l'un en saison sèche et l'autre en saison des pluies. Les blocs de Fischer complètement randomisés ont été adoptés. De manière spécifique, il s'est agit, à partir de comptages simples, de déterminer sur la parcelle après apport d'insecticides, les incidences du TYLCV, du flétrissement bactériens et des nématodes à galles sur six cultivars de tomate d'une part, et de mesurer le rendement potentiel de ces variétés comparativement à la variété Petomech cultivée en Côte d'Ivoire

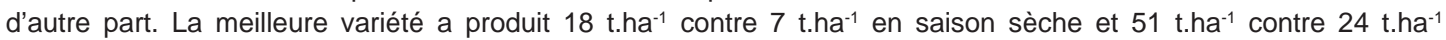
respectivement pour les variétés testées et Petomech en saison des pluies. Les résultats obtenus ont permis la classification hiérarchisée des différentes variétés, en fonction de leur origine, sur les deux cycles de production. Ainsi, les variétés d'Israël sont plus performantes que celles de Taiwan qui elles-mêmes sont plus performantes que la variété Petomech.
\end{abstract}

Mots clés : Performance, tomate, jaunisse en cuillère, flétrissement bactérien, nématodes, Côte d'Ivoire.

\begin{abstract}
Performance of six cultivars of tomato Lycopersicon esculentum Mills. against the Tomato Yellow Leaf Curl Virus (TYLCV), the bacterial wilt and the root knot nematodes

Tomato is widely produced in Côte d'Ivoire. Nonetheless, tomato production faces several constraints then whose principal ones are among others diseases: the yellow leaf curl, bacterial wilt and root knot nematodes. In order to identify performants cultivars, six improved cultivars of tomato were tested in the Toumodi region (Côte d'Ivoire). The trial was conducted over two production cycles; one in the dry season and the other one in the rainy season. The experimental design was based on the Fisher completely Randomized Blocks. Basically, we had to determine after using pesticides, the incidence of TYLCV, bacterial wilt and root knot nematodes on the six cultivars of tomato. We compared the potential yield of those varieties to that of the Petomech one. The best yield obtained is 18 t.ha ${ }^{-1}$ against 7 t.ha ${ }^{-1}$ in dry season and then, 51 t.ha ${ }^{-1}$ against 24 t.ha ${ }^{-1}$ in rainy season for the tested varieties and Petomech respectively. The results obtained allowed us to make a hierarchical classification of the cultivars according to their origin over the two cycles of production. We concluded that, the varieties of Israel are more productive than those of Taiwan, which are in turn more preferment than the Petomech one.
\end{abstract}

Key words: Performance, Tomato, yellow leaf curl, bacterial wilt, Nematodes, Côte d'Ivoire. 


\section{Introduction}

Parmi les plantes maraîchères cultivées dans le monde, la tomate est l'une des plus largement produites en plein champ et dans les jardins (Salunkhe et Kadam, 1998). La superficie de tomate plantée en 2001, s'élève à 3,7 millions d'hectares avec une production estimée à 100 millions de tonnes (Causse et al., 2003 ; FAO, 2003).

Cependant, cette production est inégalement repartie. L'Asie occupe le premier rang avec 45 $\%$ de la production mondiale, l'Europe le $2^{\text {ème }}$ rang avec $22 \%$ suivie de l'Amérique $19 \%$ et l'Afrique $12 \%$ (FAO, 2003). Tandis qu'au niveau mondial le rendement moyen est d'environ 25 t.ha $^{-1}$ (Anonyme 1, 1989 ; Messiaen et al., 2000), en Afrique au sud du Sahara il n'est que de 10 t.ha $^{-1}$ (Nono et al., 2001). En Côte d'Ivoire cette faible productivité crée un déficit structurel au niveau de l'offre des produits de la tomate (Minagra, 1993). En effet, les besoins en tomates estimés à plus de 100000 tonnes ne sont couverts qu'aux 2/3 par la production locale.

Les dégâts causés par les Hémiptères dont Bemisia tabaci (Genadius) vecteur du TYLCV (Tomato Yellow Leaf Curl Virus) et les Nématodes du genre Meloidogyne, restent de loin les plus importants et les plus dommageables pour la culture de tomates en saison sèche (Burban, 1991 ; Sawadogo et al., 2000). Le TYLCV, maladie virale qui cause l'enroulement des feuilles de tomates réduit le rendement et entraîne parfois un nanisme et une absence de fruit lorsque les attaques sont précoces (Hanafi, 2000 ; Nono et al., 2001). La lutte contre cette maladie est basée sur la réduction des populations de $B$. tabaci qui en est le vecteur (Burban, 1991; Hanafi, 2000). La tolérance des plantes a cette maladie est perdue lorsque le taux d'inoculum virale est important ou lorsque les racines des plantes sont parasitées par les nématodes du genre Meloidogyne responsables des galles (Nono et al., 2001). En plus de ces insectes et nématodes, les champignons (Stemphylium sp., Alternaria $s p$. et Fusarium $s p$.) et surtout les bactéries (Ralstonia solanacerum) causent de nombreux dégâts aux cultures de tomates en saison sèche, (Fauquet \& Thouvenel, 1987). Ces dégâts entraînent la perte d'environ 70 \% de la production si des mesures sanitaires ne sont pas prises.

La demande de tomates étant croissante et les variétés habituellement cultivées en Côte d'Ivoire plus sensibles au TYLCV, au flétrissement bactérien et aux nématodes à galles, et conséquemment moins productives, il apparaît important de rechercher des variétés de tomates mieux adaptées aux contraintes parasitaires en période de saison sèche; période pendant laquelle les prix de vente de la tomate sont très attractifs (Dao et al., 2003). La présente étude est réalisée afin d'identifier des cultivars de tomates qui, dans un système de protection phytosanitaire adéquat permettent d'obtenir de meilleurs rendements.

\section{Matériel et méthodes}

\subsection{Matériel}

\subsubsection{Site de l'étude}

L'étude a été conduite dans la zone de transition savane-forêt sur le site de recherche du Centre Suisse de Recherches Scientifiques (CSRS) à Bringakro (6,52 Nord, $-5,23$ Ouest) au centre de la Côte d'Ivoire. Cette zone est dominée par des plateaux parfois cuirassés. Les sols appartiennent à la sous-classe des sols ferralitiques moyennement et faiblement désaturés (Ettien, 2004). Les valeurs de pH comprises entre 4,5 et 6,5 , et des bases échangeables comprises entre 1 mé/100 g et 8 mé/100 g, ainsi que du taux de saturation indiquent qu'il s'agit d'un sol ferralitique moyennement saturé, induré, selon la classification de la FAO (Ettien, 2004).

\subsubsection{Matériels végétal, chimique et technique}

Le matériel végétal est constitué de six cultivars de la tomate Lycopersicon esculentum Mills. provenant de trois origines : deux variétés d'Israël ( $T$ 3331, T 3328), trois variétés de Taiwan (CLN 2026 D4, CLN 2443 A et CLN 2443 B) et une variété locale qui a servi de témoin (Petomech) (Tableau 1).

Le matériel chimique est constitué d'insecticides (Imidacloprid, Biprofezim, et Abamectine), de fongicides (Chlorothalonil, Carbendazime, et Mancozèbe), d'un bactéricide (Oxyde de cuivre) et d'un nématicide (Carbofuran).

Un système d'irrigation goutte à goutte de type FDS (Family drips system, NETAFIM, Israël) était utilisé pour les apports d'eau. 
Tableau 1 : Origine du matériel végétal et quelques caractéristiques agronomiques fournies par les sélectionneurs.

\begin{tabular}{|c|c|c|c|c|c|}
\hline Variétés & Origines & $\begin{array}{c}\text { Adaptation aux } \\
\text { hautes températures }\end{array}$ & poids par fruit (g) & $\begin{array}{l}\text { Résistance } \\
\text { Tolérance }\end{array}$ & $\begin{array}{c}\text { Rendement } \\
\text { potentiel }\left(\mathrm{t} . \mathrm{ha}^{-1}\right)\end{array}$ \\
\hline Petomech & $\begin{array}{c}\text { Côte } \\
\text { d'Ivoire }\end{array}$ & $N / D$ & 80 & N/D & N/D \\
\hline T 3328 & Israël & ** & 80 & $\begin{array}{c}\text { V, F1-2, T, Ty, N, } \\
\text { S, C, }\end{array}$ & 140 \\
\hline T 3331 & Israël & ** & $80-110$ & $\begin{array}{c}\text { V, F1-2, T, Ty, N, } \\
\text { S, C, }\end{array}$ & 140 \\
\hline CLN 2026 D4 & Taiwan & $\mathrm{N} / \mathrm{D}$ & $80-100$ & $\begin{array}{c}\text { Ty, Ft, Alte F1,2, } \\
\text { V, N, S, }\end{array}$ & $N / D$ \\
\hline CLN 2443 A & Taiwan & $N / D$ & $80-90$ & $\begin{array}{c}\text { Ty, Ft, Alte F1,2, } \\
\text { V, N, S, }\end{array}$ & $\mathrm{N} / \mathrm{D}$ \\
\hline CLN 2443 B & Taiwan & N/D & $80-90$ & $\begin{array}{c}\text { Ty, Ft, Alte F1,2, } \\
\text { V, N, S, }\end{array}$ & $\mathrm{N} / \mathrm{D}$ \\
\hline
\end{tabular}

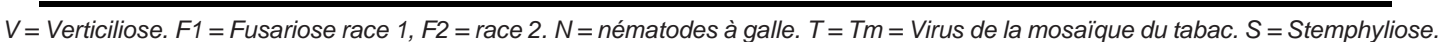
$C=$ Cladosporiose. $T y=$ Virus de la jaunisse en cuillère des feuilles de tomate.. Ft $=$ Flétrissement bactérien. $N / D=n o n$ déterminé. ${ }^{\star *}=$ Bonne adaptation

Sources : Israël (fiche accompagnant les semences, www.hazera.co.il); Taiwan (fiche accompagnant les semences, www.avrdc.org).

\subsection{Méthodes}

La parcelle utilisée pour les expérimentations avait pour précédent cultural la tomate et caractérisée par une forte pression d'infestation en ce qui concerne les nématodes du genre Meloidogyne, les bactéries (Ralstonia solanacerum pv. Vesicatoria) et les insectes ( $B$. tabaci). Les produits phytosanitaires sont utilisés selon le chronogramme suivant:

l'Imidacloprid est utilisé 15 jours après le semis en pépinière, en arrosage au pied des plantes et en cours de végétation, 35 jours après semis à la dose de 1,2 litres par hectare.

le carbofuran (nématicide) est apporté en pépinière juste avant la mise en terre des graines de tomate puis au repiquage par épandage sur les lignes de plantation à la dose de $10 \mathrm{~g} . \mathrm{m}^{-2}$ en pépinière et $10 \mathrm{~g}$ par mètre linéaire au repiquage. Après le repiquage, les insecticides (Biprofezim et Abamectine), les fongicides (Chlorothalonil et Carbendazime) et le bactéricide (Oxyde de cuivre), sont apportés en pulvérisation foliaire une fois tous les 15 jours. Les doses de 1,2 litres, 2 litres par hectare sont apportés respectivement pour les insecticides et les fongicides puis, la dose de $2 \mathrm{~kg}$ par hectare pour le bactéricide à 35, 50 et 65 jours.

Cette étude est réalisée sur deux cycles de culture, l'un en saison sèche et l'autre, en saison des pluies.

Le dispositif expérimental est réalisé en blocs de Fischer avec 4 répétitions. Chaque parcelle élémentaire de $12 \mathrm{~m}^{2}$ est plantée à la densité de 33333 plants par hectare.

Concernant la maladie de TYLCV, les observations ont consisté en un dénombrement des plants présentant les symptômes de jaunisse en cuillère, du repiquage au $55^{\mathrm{e}}$ jour (feuilles jeunes jaunes, limbe réduit, recroquevillé vers le haut, réduction des entre-nœuds, réduction générale de la croissance de la plante). Concernant le flétrissement bactérien (fanaison progressive du haut vers le bas des feuilles de la plante à l'état chlorophyllienne), le nombre de plantes atteintes est relevé tous les deux jours, puis déterrées et les racines observées, pour dénombrement des galles dues aux nématodes. Pour le TYLCV, les feuilles jeunes des plantes sont observées toutes les deux semaines. Au cours des observations, les plantes infectées sont aussi déterrées pour observer les galles 
dues aux nématodes. Le cumul des plantes éliminées à cause de l'une de ces maladies jusqu'au $55^{\text {ème }}$ jours après repiquage a servi d'indicateur de jauge de la sévérité des dégâts liés aux attaques.

Quant à l'incidence des nématodes, à la fin de la récolte, toutes les plantes sont déterrées et cotées ; 1 (présence de galles) et 0 (plant sain sans galles).

Pour l'analyse des résultats, un modèle GLM (General Linear Model) du logiciel SAS est utilisé, et les comparaisons des moyennes sont réalisées au seuil de $5 \%$ de probabilité.

\section{Résultats}

\subsection{Maladies}

Le taux des plantes présentant les symptômes du TYCLV varie entre 4,6 et $14,4 \%$ en saison sèche (Tableau 2). En saison des pluies, il varie entre 1,9 et 8,7 \%. En saison sèche, seules les variétés Petomech et T 3328 se distinguent des autres variétés. En saison des pluies, les analyses statistiques ne permettent pas de séparer la variété Petomech de la variété 2443 A.

Le taux moyen d'infestation pour le flétrissement bactérien des variétés améliorées (Israël et Taiwan) en saison sèche est significativement inférieur à celui de la variété locale, 10 et $20 \%$ contre $32 \%$. Au sein des variétés améliorées, I'on peut distinguer trois groupes. Le groupe B formé par les variétés CLN 2026 D4 et T 3328. le groupe C est formé par la variété CLN 2343 A, et enfin le groupe $D$, formé par la variété T 3331. La variété T 3331 montre un taux d'infestation plus faible que toutes les autres (5\%).

En saison des pluies, la variabilité du flétrissement bactérien est également forte et elle permet selon le test de DUNCAN de regrouper les variétés par origine.

Au niveau de la galle des nématodes, le taux d'attaque est variable sur les variétés au cours des deux cycles de production (Tableau 2). Elle est forte en saison des pluies pour T 3331 et $T$ 3328 respectivement de 71 et $79 \%$. Puis, faible en saison sèche respectivement de 1,3 et 9,5 \% De même, comme pour les deux autres maladies étudiées ci-dessus, aucun groupe de variétés d'après leur origine ne se distingue des autres en saison sèche (Tableau 2). Par contre, en saison des pluies, les variétés T 3331 et T 3328 ont des niveaux d'incidences très prononcés concernant la galle des nématodes. Cette distinction permet au cours de cette saison, d'obtenir deux groupes de variétés. Celui formé par les deux variétés d'Israël et celui constitué par les variété Petomech et celles de Taiwan.

\subsection{Les rendements}

Le modèle linéaire général (GLM) est significatif au seuil de $5 \%$ et permet d'expliquer $90 \%$ de la variabilité du rendement entre les différentes variétés en saison sèche et $87 \%$ de la variabilité en saison des pluies. Quel que soit le cycle, toutes les variétés améliorées ont en moyenne un rendement supérieur à celui de Petomech (Tableau 2), mais entre les trois différentes origines de variétés, seule la variété CLN 2026 D4 se distingue des autres. En saison sèche, les variétés sont regroupées en quatre classes distinctes. La classe a, formée par T 3331 et T 3328 avec un rendement de 18 t.ha $^{-1}$. La classe ab est formée par les lignées fixées CLN 2443 B et CLN 2443 A avec des rendements intermédiaires d'environ 16 t.ha $^{-1}$, mais indistincts des deux premières (hybrides). La classe $c$ est formée par la variété CLN 2026 D4 avec un rendement de 10 t.ha $^{-1}$. La variété Petomech avec un rendement de 7 t.ha $^{-1}$ forme la classe d (Tableau 2).

En saison des pluies, le test de DUNCAN permet de regrouper les variétés en trois groupes. Le groupe a, formé par les variétés d'Israël, le groupe $b$ formé par les variétés de Taiwan et le groupe c constitué de la variété locale Petomech. Les rendements varient en générale de 24 à 51 t.ha $^{-1}$ (Tableau 2). Au niveau des classes, la plus petite différence significative entre deux classes est de 10 t.ha $^{-1}$. Les contrastes montrent les relations qui existent entre la saison, les blocs, les maladies et les rendements des variétés (Tableau 3). 
Tableau 2 : Incidence des maladies de la tomate Lycopersicon esculentum Mills. jusqu'au 55e jour après semis et les rendements potentiels

\begin{tabular}{|c|c|c|c|c|c|}
\hline Cycle cultura & & & Maladies & & Productivité \\
\hline Saison & Variétés & TYLCV \% & $\begin{array}{l}\text { Flétrissement } \\
\text { bactérien } \%\end{array}$ & Galle \% & $\begin{array}{l}\text { Rendement } \\
\text { t.ha-1 }\end{array}$ \\
\hline \multirow{6}{*}{ 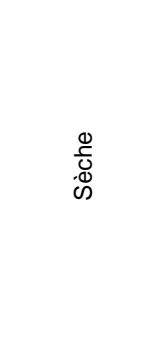 } & CLN 2026 D4 & $8,1 \mathrm{bc}$ & $25 b$ & $23,8 \mathrm{a}$ & $10,6 \mathrm{c}$ \\
\hline & CLN 2443 A & $10 a b$ & $16,3 \mathrm{c}$ & $5,6 \mathrm{~b}$ & $15 a b$ \\
\hline & CLN 2443 B & $5,6 \mathrm{bc}$ & $20 \mathrm{bc}$ & $20,6 \mathrm{a}$ & $16,3 a b$ \\
\hline & Petomech & $14,4 \mathrm{a}$ & $32,5 \mathrm{a}$ & $20,6 \mathrm{a}$ & $7 d$ \\
\hline & Т 3328 & $4,6 \mathrm{c}$ & $24,3 \mathrm{~b}$ & $9,5 \mathrm{~b}$ & $18, a$ \\
\hline & Т 3331 & $7,5 \mathrm{bc}$ & $5 d$ & $1,3 \mathrm{c}$ & $18,5 \mathrm{a}$ \\
\hline \multirow{6}{*}{$\frac{\frac{\omega}{J}}{\frac{\partial}{\alpha}}$} & CLN 2026 D4 & $1,9 \mathrm{c}$ & $5,8 \mathrm{a}$ & $19 \mathrm{~b}$ & $37,6 \mathrm{~b}$ \\
\hline & CLN 2443 A & $7,2 \mathrm{a}$ & $1,9 \mathrm{c}$ & $17 \mathrm{~b}$ & $38,9 \mathrm{~b}$ \\
\hline & CLN 2443 B & $5,3 \mathrm{~b}$ & $6,7 \mathrm{a}$ & $12 \mathrm{~b}$ & $35 \mathrm{~b}$ \\
\hline & Petomech & $8,7 \mathrm{a}$ & $5,8 \mathrm{a}$ & $19 \mathrm{~b}$ & $24 \mathrm{c}$ \\
\hline & T 3328 & $2,9 \mathrm{c}$ & $3,8 \mathrm{~b}$ & $79 a$ & $51,3 a$ \\
\hline & Т 3331 & $2,4 \mathrm{c}$ & $3,8 \mathrm{~b}$ & $71 \mathrm{a}$ & $49,6 \mathrm{a}$ \\
\hline
\end{tabular}

Les rendements, $R^{2}=90 \%$ en saison sèche et $R^{2}=87 \%$ en saison des pluies.

Les valeurs suivies de la même lettre pour la même saison, ne sont pas significativement différentes d'après le test de DUNCAN.

Tableau 3 : Tableau synoptique des effets du modèle statistique sur les variétés et les contrastes (erreurs résiduelles) au cours des deux cycles

\begin{tabular}{cc}
\hline Les effets du modèle sur le rendement réel des variétés en fonction des saisons \\
\hline Modèle général & $\mathrm{R}^{2}=0,87$, pour $\mathrm{F}(\mathrm{p})<0,0001$ \\
Variété & 0,0001 \\
Saison & 0,0001 \\
variété*saison & 0,4692 \\
Bloc (saison) & 0,0016 \\
En saison sèche & $\mathrm{R}^{2}=0,61$, pour $\mathrm{F}(\mathrm{p})<0,0001$ \\
Variété & 0,0219 \\
Bloc & 0,2031 \\
Petomech-autres variétés & 0,0086 \\
Taiwan-Israël & 0,0175 \\
En saison des pluies & $\mathrm{R}^{2}=0,77$, pour $\mathrm{F}(\mathrm{p})<0,0001$ \\
Variété & 0,0061 \\
Bloc & 0,0018 \\
Petomech-autres variétés & 0,0025 \\
Taiwan-Israël & 0,006 \\
\hline
\end{tabular}




\section{Discussion}

Les intensités de la jaunisse des feuilles ont diminué sur le site en saison des pluies. Ce résultat a été obtenu dans le centre de la Côte d'Ivoire au Burkina et au Maroc dans des études en station au niveau de la dynamique de Bemisia tabaci vecteur du TYLCV (Anonyme 2, 1996 ; Hanafi, 2000 ; Gnankiné et al., 2007). Mais, au niveau de la galle sur les variétés de Israël, c'est plutôt pendant cette période que les taux les plus élevés ont été constatés. Une analyse de la variation des différents contrastes au niveau des effets variétés, saison, bloc, Petomech-autres variétés et AVRDC-Hazera au niveau des deux cycles de la culture a permis de mieux comprendre les facteurs intervenant dans l'expression du rendement (Hassan et al., 2000).

Ainsi, concernant le TYLCV, la saison et la variété elle-même sont les deux variables qui ont un effet sur l'apparition de la maladie chez la plante (Hassan et al., 2000). Par contre, tous les effets du modèle permettent d'expliquer l'action des bactéries responsables du flétrissement bactérien sur le site, sauf l'interaction bloc saison qui n'est pas significatif $F(p)<0,07$. Ce résultat était attendu car la bactérie responsable du flétrissement (Ralstonia solanacerum) étant terricole, il n'y a pas de migration particulière dans les différents blocs en fonction des saisons, ni d'une action particulière de la saison sur les bactéries d'un bloc à un autre (Pilowsky \& Zutra, 1986 ; Opeòa et al., 1989). L'action des bactéries est liée aux températures du milieu et à leur biologie particulière (Nono et al., 2001). C'est ce qui explique les différents effets significatifs. II en est de même pour la galle des nématodes où l'interaction bloc - saison n'est pas significatif $F(p)<0,1$. Les taux d'infestations obtenus en saison des pluies sur les variétés T 3328 et T3331 au niveau de la galle des nématodes et du TYLCV sont en contradiction avec ceux obtenus par d'autres auteurs dans les mêmes conditions d'infestation (Girard et al., 1989 ; Mateille, 1994). Pour ces auteurs, lorsque les plantes sont parasitées par les nématodes, elles deviennent très sensibles au TYLCV, ce qui n'est pas le cas des variétés étudiées dans ce travail. Mais au niveau des infestations dues aux nématodes, ces résultats sont bien conforment pour une plante sensible. En effet, la saison des pluies constitue avec l'humidité élevée du sol et des variations de températures, la période favorable a la pullulation des nématodes.

Les différences entre les rendements sont expliquées par la saison et les variétés. Mais l'interaction (saison-variété) n'a aucune influence ; ce qui permet de dire que la saison agit de la même manière sur toutes les variétés au cours des différents cycles de culture (Girard et al., 1989 ; Saliba et al., 2000). Ainsi, si une différence est observée entre les variétés au cours d'une même saison, elle est due à la variété seule.

En considérant le cas de la Côte d'Ivoire, les rendements potentiels obtenus sont au-dessus de la moyenne nationale, 10 t.ha $^{1}$ (Minagra, 1993 ; Nono et al., 2001). Par rapport à l'essai réalisé en saison sèche, celui de la saison pluvieuse permet la confirmation des performances des variétés en termes de rendement potentiel.

Les comparaisons des variétés entre elles en fonction de leur origine permettent de dire que la variété Petomech prise comme témoin dans l'essai est différente des autres variétés qui sont elles-mêmes différentes entre elles, en fonction de leur origine. Mais l'analyse des rendements entre les variétés d'une même origine ne permet pas de dire qu'elles sont différentes entre elles à travers les différentes saisons. De ce fait, un classement possible en fonction de la performance des variétés liée aux résultats obtenus ne peut se faire que par origine.

\section{Conclusion}

La saison des pluies reste celle favorable à la production de tomates parce que les principales maladies, à savoir le TYLCV et le flétrissement bactérien sont moins importantes en cette période. La saison sèche est moins favorable à cause du parasitisme très élevé. Compte tenu de la sensibilité de la variété Petomech et les performances des variétés d'Israël et de Taiwan, il serait pertinent de remplacer cette première cité sur le terrain par les variétés plus performantes. Cependant, vu la sensibilité des variétés d'Israël, l'utilisation de nématicide en prétraitement avant les plantations pourrait améliorer d'avantage leur productivité.

Concernant les performances des variétés face aux maladies, les analyses n'ont pas permis de faire une classification hiérarchique des variétés prises individuellement ou par origine. Cependant, il ressort que les variétés d'Israël et 
de Taiwan sont capables de donner de hauts rendements lorsque des pesticides sont apportés au cours de la production. En ce qui concerne les performances des variétés en termes de rendements, les analyses permettent de les classer par origine.

\section{Références citées}

Anonyme 1, 1989. Tomato and Pepper production in the tropics. International symposium on integrated management practices, AVRDC, Tainan, Taiwan 21- 26 March 1989, 586 pp.

Anonyme 2, 1996. Institut des Savanes (IDESSA), Rapport d'activités du programme de recherche sur la tomate, PRG/ TRITURAF, Bouaké (Côte d'Ivoire), $90 \mathrm{pp}$.

Burban C., 1991. Structuration des populations chez un insecte polyphage: Bemisia tabaci Gennadius) (Homoptera : Aleyrodidae). Thèse de Doctorat d'Etat, Université des sciences et techniques du Languedoc, Montpellier, 100 pp.

Causse M., Buret M., Robini K. \& Verschave P., 2003. Inheritance of nutritional and sensory quality traits in fresh market tomato and relation to consumer preferences. J. of Food Sci., 68 (7): 2342-2350.

Dao D., Kouakou A., Cissé G., \& Girardin O., 2003. Rapport d'activités de la phase pilote du PCVI (Programme des Cultures Vivrières Intensives) 1999-2002, Zatta (Yamoussoukro), Tiémélékro (Dimbokro), CSRS, 01 BP 1303 Abidjan, Côte d'Ivoire, 76 pp.

Ettien D.J.B., 2004. Intensification de la production d'igname (Dioscorea spp.) par la fertilisation minérale et l'identification de nouvelles variétés en zone forestière et savanicole de Côte d'Ivoire. Thèse de Doctorat Unique en Sciences de la Terre option: Agro Pédologie. Université de Cocody (Côte d'Ivoire) 187 pp.

FAO., 2003. La production de tomate. Dans les média demain 6-817, 1-6.

Fauquet C., \& Thouvenel J., 1987. Maladies virales des plantes en Côte d'Ivoire, ORSTOM, Paris, 82 pp.

Girard J.-C., Marchand J. L., \& Michellon M., 1989. Search for bacterial wilt resistant tomato varieties in reunion. In: Anonym. Eds. AVRDC. International symposium on integrated management practices, Tainan, Taiwan 21- 26 March 1989, pp. 228-230.
Gnankiné O., Traoré D., Sanon A., Traoré N.S., \& Ouédraogo A.P., 2007. Traitements insecticides et dynamique des populations de Bemisia tabaci Gennadius en culture cotonnière au Burkina Faso, Cah. Agri. 16 (2) : 101-109.

Hanafi A., 2000. La mouche blanche et le virus des feuilles en cuillère de la tomate. Bulletin mensuel d'information et de liaison du PNTTA. 73 (4) : 6-12.

Hassan A. A., Laterrot H., Mayzad H. M., \& Nakhla M. K., 2000. Use of Lycopersicon peruvianum as a source of resistance to Tomato yellow leaf curl virus. Egypt. J. Hortic., 14 (2) : 173-176.

Mateille T., 1994. Biologie de la relation plantesnématodes: perturbations physiologiques et mécanismes de défense des plantes. Nematologica, 40 : 276-311.

Messiaen J-C., 1989. Environmental influences on the severity of tomato bacterial wilt in the French West Indies. Interactions with varietal resistance. In: Green, S. K \& McAllen, B. M., Eds. AVRDC. Shanhua, Taiwan, pp. 234-236.

Messiaen C. M., Migliori A., \& Maison P., 2000. Effet de la Mosaïque du tabac (TMV) sur la croissance et la fructification des cultures de tomate de plein champ dans le Sud-est de la France. Etude de Virologie. Ann. Epihyt., 19 (4) : 93-102.

Minagra, 1993. Plan directeur du développement agricole 1992-2015. Ministère de l'agriculture, Abidjan, République de Côte d'Ivoire 257 pp.

Nono W.R., Swai I. S., \& Chadha M. L., 2001. Management of vegetable diseases in Eastern and Southern Africa: case study of tomato. In: Anonym, Proceedings of the workshop on vegetable research and development in West Africa. Eds. AVRDC Africa Regional Program, ARUSHA TANZANIA. pp. 19-31.

Opena R.T., Green S. K., Talekar N. S. \& Chen J. T., 1989. Genetic improvement of tomato adaptability to the tropics: progress and future prospects. In: Green, S. K \& McAllen, B. M., Eds. AVRDC. Shanhua, Taiwan. pp. 597-619.

Pilowsky M., \& Zutra D., 1986. Reaction of different tomato genotypes to the bacterial speck pathogen (Pseudomonas siryngae p. v. tomato) Phytoparasitica 14 (1): 39-42.

Saliba C.V., Causse M., Gervais L. \& Philouze J., 2000. Efficiency of RFLP, RAPD, and AFLP markers for the construction of an intraspecific map for the tomato genome. Genome 43 (1): 29-40. 
Salunkhe D.K., \& Kadam S.S., 1998. Handbook of vegetable science and technology, Production, composition and processing. Department of cellular biology and technology, University of Auckland, New Zealand. 203 pp.
Sawadogo A., Diop M.T., Thio B., Konate Y.A., \& Mateille T., 2000. Incidence de quelques facteurs agronomiques sur les populations de Meloidogyne spp. et leurs principaux organismes parasites en culture maraichère sahélienne, Nematology, 2 : 895-906. 